\title{
Current Policies for Surveillance and Management in Women at Risk for Breast and Ovarian Cancer: A Survey Among 16 European Family Cancer Clinics
}

\author{
H.F.A. Vasen, N.E. Haites, D.G.R. Evans, \\ C.M. Steel, P. Møller, S. Hodgson, \\ D. Eccles, P. Morrison, D. Stoppa-Lyonnet, \\ J. Chang-Claude, M. Caligo and the \\ European Familial Breast Cancer \\ Collaborative Group
}

\section{INTRODUCTION}

The recent isolation of breast cancer predisposing genes (BRCAl and BRCA2) allows for the identification of carriers within affected families. These carriers have a 50 $85 \%$ risk of developing breast or ovarian cancer and need careful follow up. The purpose of this study was to evaluate the management and screening protocols implemented in high risk families at various family cancer clinics in Europe.

\section{METHODS}

A questionnaire was mailed to the members of the European Familial Breast Cancer Collaborative group requesting information on the following issues: indication for surveillance of breasts and ovaries, the recommended protocol, coordination of the screening examination, prophylactic surgery, the specific management of breast cancer in a mutation carrier and the use of estrogen.

\begin{abstract}
RESULTS
Most centres recommend surveillance of the breasts if the lifetime risk exceeds $15-20 \%$. The surveillance protocol that is generally advised comprises monthly self breast examination, examination by a specialist every six months and annual mammography, all starting from an age between 25 and 35 years. Surveillance of the ovaries is recommended in BRCA1- and BRCA2mutation carriers, in members from breast/ovarian cancer families and in some centres in - breast cancer only - families with an early onset of breast cancer. The recommended protocol includes gynaecological examination, sonography and estimation of CA-125 at yearly intervals starting from age 30-35. Prophylactic mastectomy is considered for proven mutation carriers in some centres. Most centres consider prophylactic oophorectomy in mutation carriers and some centres also consider it for members of breast/ovarian cancer families.
\end{abstract}

\section{CONCLUSION}

This survey provides insight into the guidelines for surveillance and management of familial breast cancer used at various family cancer clinics in Europe; this insight may contribute to the appropriate management of these high-risk women. It should be emphasized that most recommendations are based on expert opinion rather than on any specific studies. 